\title{
Global Expression Profiles in Human Normal and Aneurysmal Abdominal Aorta Based on Two Distinct Whole Genome Microarray Platforms
}

\author{
GUY M. LENK,${ }^{a}$ GERARD TROMP,${ }^{a}$ MAGDALENA SKUNCA,${ }^{a}$ \\ ZORAN GATALICA, ${ }^{c}$ RAMON BERGUER, ${ }^{d}$ \\ AND HELENA KUIVANIEMI ${ }^{a, b}$ \\ ${ }^{a}$ Center for Molecular Medicine and Genetics, Wayne State University School \\ of Medicine, Detroit, MI, USA \\ ${ }^{b}$ Department of Surgery, Wayne State University School of Medicine, Detroit, \\ MI, USA \\ ${ }^{c}$ Department of Pathology, Creighton University School of Medicine, Omaha, \\ NE, USA \\ ${ }^{d}$ Department of Surgery, University of Michigan, Ann Arbor, MI, USA
}

\begin{abstract}
Abdominal aortic aneurysms (AAA) are the thirteenth cause of death in the United States. The etiology of the disease is yet largely unknown, although several environmental risk factors (e.g., smoking) have been identified and the search for finding genetic risk factors has been initiated. The purpose of our study was to gain insight into the pathobiology of AAA by determining which genes are expressed in the abdominal aorta under either the diseased or normal states, thereby generating the whole-genome-wide expression profiles for these conditions.
\end{abstract}

KEYWORDS: abdominal aortic aneurysm; gene expression; genomics; pathway analysis; gene ontology

We used two distinct microarray platforms to generate global gene expression profiles to analyze the transcriptome of both aneurysmal ${ }^{1-3}$ and nonaneurysmal abdominal aorta. Aortic tissue samples were collected from patients from the following four groups: (a) male abdominal aortic aneurysm (AAA) patients $(n=4)$; (b) male autopsy controls $(n=4)$; (c) female AAA patients $(n=3)$; and $(\mathrm{d})$ female autopsy controls $(n=3)$. Case and control

Address for correspondence: Helena Kuivaniemi, M.D., Ph.D., Center for Molecular Medicine and Genetics, Wayne State University School of Medicine, 3317 Gordon H. Scott Hall of Basic Medical Sciences, 540 E. Canfield Ave., Detroit, MI 48201, USA. Voice: 313-577-8733; fax: 313-577-5218. e-mail: kuivan@sanger.med.wayne.edu

Ann. N.Y. Acad. Sci. 1085: 360-362 (2006). (C) 2006 New York Academy of Sciences. doi: 10.1196/annals.1383.041 
groups were matched for age and ethnicity. RNA was isolated from abdominal aortic tissues, labeled, and hybridized to two different whole genome microarray platforms (Affymetrix and Illumina). The samples were run as pools (both platforms) and also as individual samples (Illumina only). The results were analyzed using the statistical program R (version 2.0; Bioconductor package), GeneSpring (version 7.2), and BeadStudio (version 1.5.0.34). Cluster (version 3.0) was used for hierarchical clustering of genes with expression differences between the cases and controls and visualized with TreeView (version 1.0.12). The online database PANTHER was used to annotate the genes into ontological categories. ${ }^{4}$

The number of unique genes shared between the Affymetrix and Illumina platforms is 18,037 , representing approximately $75 \%$ of the current estimate for the number of genes in the human genome. Of the genes in this common set, 8,763 were concordant on both platforms and expressed in either normal or aneurysmal tissue. Several gene ontological categories were significantly different from that expected by chance such as "protein metabolism and modification" (overrepresented; $P=1.87 \times 10^{-8}$ ), "cell communication" (underrepresented; $P=3.04 \times 10^{-13}$ ), and "intracellular protein traffic" (overrepresented; $P=9.05 \times 10^{-10}$ ).

Differential gene expression was also examined using a fold difference threshold set to four. It was necessary to use a fold difference measure due to limited samples on the Affymetrix platform. A total of 468 unique genes, out of the common gene set, met the criterion in both array platforms. Several of the genes in this list have been reported previously as being possibly important in AAA formation or growth such as MMP $1,{ }^{5} \mathrm{MMP} 9,{ }^{6}$ and IL $1 \mathrm{~B}^{7}$ to name a few. Analysis of the gene ontological categories of these differential genes showed that the Biological Process category "immunity and defense" was extremely overrepresented $\left(P=2.04 \times 10^{-20}\right)$ as was "cell communication" $(P=2.74 \times$ $\left.10^{-12}\right)$ and "cell structure and motility" $\left(P=9.77 \times 10^{-8}\right)$.

In conclusion, these data provide global expression profiles for normal and aneurysmal abdominal aorta, and point to the importance of immune defense and tissue remodeling in the course of aneurysmal disease. Knowing which genes are actively transcribed in either normal or aneurysmal aorta is useful in identifying plausible positional candidate genes for diseases of the aorta. Thus, the insights provided by these data are valuable for future research and will aid in the discovery of therapeutic targets for the treatment of AAAs.

\section{ACKNOWLEDGMENTS}

The study was supported in part by a grant from NHLBI (HL064310). Guy M. Lenk is a Pre-Doctoral Fellow of the American Heart Association. 


\section{REFERENCES}

1. ERnst, C.B. 1993. Abdominal aortic aneurysms. N. Engl. J. Med. 328: 1167-1172.

2. Lederle, F.A., G.R. Johnson, S.E. Wilson, et al. 2000. The aneurysm detection and management study screening program: validation cohort and final results. Aneurysm Detection and Management Veterans Affairs Cooperative Study Investigators. Arch. Intern. Med. 160: 1425-1430.

3. Shibamura, H., J.M. Olson, C. van Vlijmen-Van Keulen, et al. 2004. Genome scan for familial abdominal aortic aneurysm using sex and family history as covariates suggests genetic heterogeneity and identifies linkage to chromosome 19q13. Circulation 109: 2103-2108.

4. Mi, H., B. Lazareva-Ulitsky, R. Loo, et al. 2005. The PANTHER database of protein families, subfamilies, functions and pathways. Nucleic Acids Res. 33: D284-D288.

5. Irizarry, E., K.M. Newman, R.H. Gandhi, et al. 1993. Demonstration of interstitial collagenase in abdominal aortic aneurysm disease. J. Surg. Res. 54: 571-574.

6. Newman, K.M., Y. Ogata, A.M. Malon, et al. 1994. Identification of matrix metalloproteinases 3 (stromelysin-1) and 9 (gelatinase B) in abdominal aortic aneurysm. Arterioscler. Thromb. Vasc. Biol. 14: 1315-1320.

7. Pearce, W.H., I. SweIS, J.S. Yao, et al. 1992. Interleukin-1 beta and tumor necrosis factor-alpha release in normal and diseased human infrarenal aortas. J. Vasc. Surg. 16: 784-789. 